\title{
CULEX BRUMPTI, N. SP. MOUSTIQUE NOUVEAU TROUVÉ EN CORSE
}

\author{
Par H. GALliARD
}

Dans une note parue en 1927 dans ces Annales (1), j'avais signalé la présence, en Corse, dans deux localités (lit du Tavignano près d'Aléria, et Campo di Loro près d'Ajaccio) de larves trouvées en 1925, qui différaient à première vue, en raison du caractère très particulier des dents du peigne du siphon, de toutes celles décrites dans la région paléarctique. J'avais discuté les autres caractères qui rappelaient un peu ceux des larves à long siphon avec touffes de soies rares et très courtes : Culex tritæniorhynchus Giles, Culex nerexiguus Theobald (C. univittatus Theobald) et Culex laurenti Newstead. Je l'avais provisoirement rapportée à $C$. perexiguus.

En août 1928, j'ai pu retrouver ces mêmes larves dans le gîte du Campo di Loro et, par élevage, obtenir des adultes, deux mâles et deux femelles dont la description est donnée ci-dessous.

\section{Larve}

Je rappellerai les principaux caractères de la larve : elle est d'assez grande taille, plus grande que la larve au $4^{\circ}$ stade de C. univittatus. Les antennes présentent une touffe de soies située à l'union du tiers antérieur et du tiers moyen. Soies péristomales externes et antérieures à deux branches, externes et postérieures à trois branches, une paire de soies médianes courtes et/grêles. Touffes de soies préantennaires à 8 branches plumeuses. Plaque mentale à 13 dents, 6 de chaque côté de la dent centrale, les deux externes plus petites et séparées des autres.

Abdomen. - Le peigne du $8^{\circ}$ segment est formé d'environ 40 dents à extrémité mousse et finement denticulées sur les bords jusqu’à la base. Le segment anal ne présente rien de particulier : les branchies sont de la même longueur que le bord dorsal de la manchette chitineuse.

(1) Note sur les Culicinés de Corse. Annales de Parasitologie, V, 1927, p. 97. AnNales de Parasitologie, T. IX, $\mathrm{N}^{\circ} 2 .-1^{\mathrm{er}}$ mars 1931 p. 134-139. 

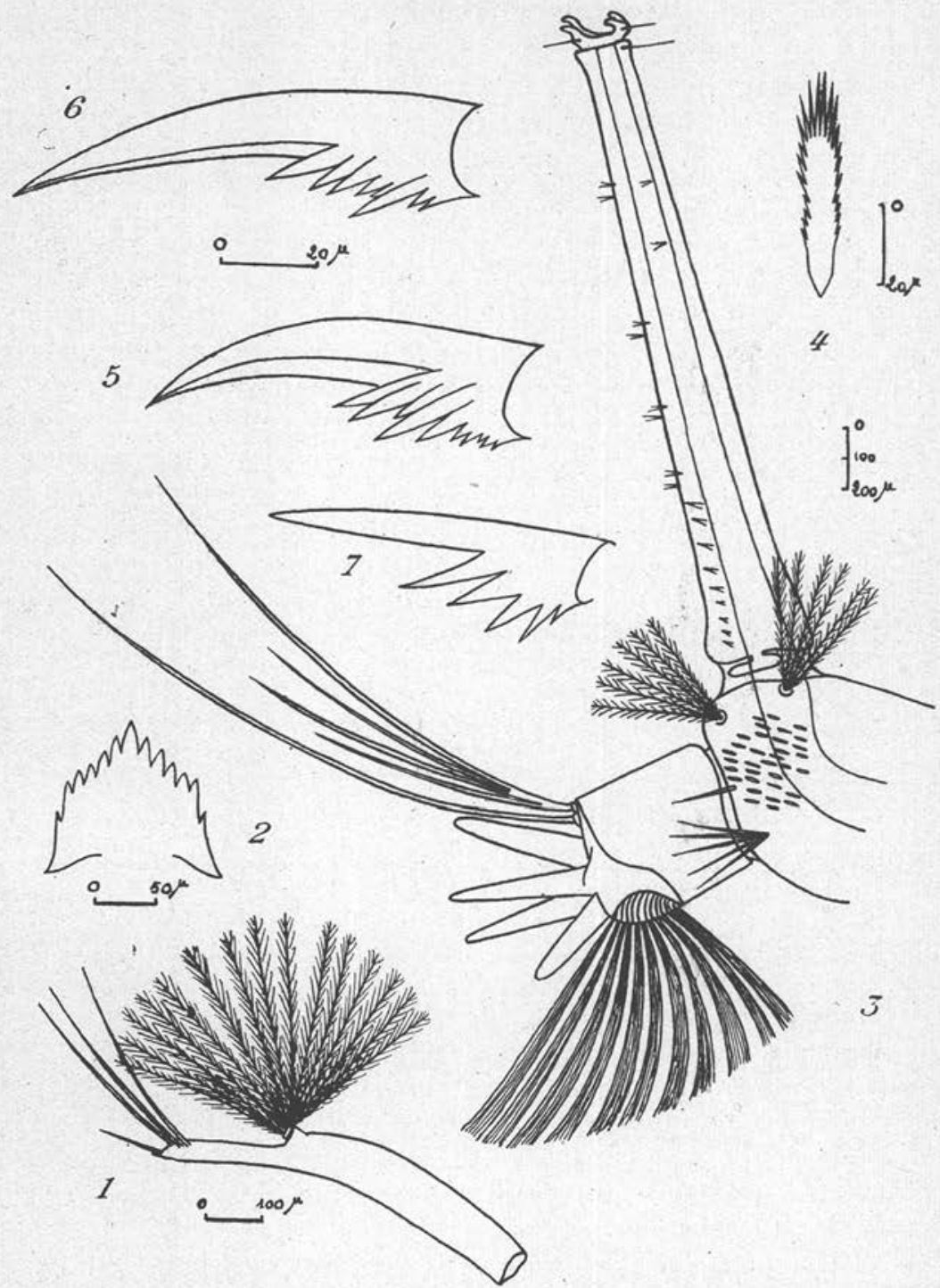

Fig. 1. - Larve de Culex brumpti. 1, antenne; 2, plaque mentale; 3, siphon et segment anal ; 4 , une dent du 8 e segment ; 5 et 6 , dernière et avant-dernière dent du peigne du siphon ; $7,5^{\mathrm{e}}$ dent du peigne.

Siphon. - Coloration foncée. Indice 6. Six paires de touffes de soies extrêmement courtes et grêles, beaucoup plus courtes que la 
largeur du siphon à ce niveau. Quatre paires sont ventrales, la première située un peu en avant de la dernière dent du peigne ; les trois premières sont équidistantes, la quatrième est nettement séparée et située près de l'apex du siphon; deux paires latérales situées dans l'intervalle compris entre les $3^{\circ}$ et $4^{\circ}$ paires ventrales.

Peigne du siphon. - Formé de 9 à 10 dents. Les premières près de la base sont rapprochées, les autres s'écartent progressivement les unes des autres, la dernière est située au tiers du siphon. Les quatre ou cinq dernières sont remarquablement fortes et grandes. Tout à fait à leur base sont groupées 10 à 12 épines très rapprochées et de taille variable. Ces dents sont tout à fait caractéristiques. Il n'est pas possible de confondre cette larve avec celles de $C$. tritæniorhynchus, dont les dents du siphon présentent des épines nombreuses s'étendant jusqu'à l'apex, ni avec celles de $C$. univittatus, dont ces mêmes dents ne présentent aucune particularité remarquable. A ma connaissance, parmi les larves de Culex de la région paléarctique, seuls $C$. laticinctus et surtout $C$. theileri en présentent de semblables, mais les autres caractères sont suffisamment typiques pour éviter toute erreur.

\section{Adulte}

Les spécimens obtenus par élevage montrent que les adultes normaux doivent être d'assez grande taille. Sur le sommet de la tête on trouve des écailles noirâtres dressées verticalement et des écailles claires; autour des yeux, une mince bordure d'écailles blanches plus grandes et qui s'épaissit sur les côtés de l'occiput.

Trompe. - Labelles clairs ; quart antérieur de la face inférieure - sombre. Sur la partie moyenne, écailles claires s'étendant presque jusqu'à la base, la limite postérieure de cette zone claire étant moins bien définie que l'antérieure, ces écailles débordant à peine sur la face supérieure.

Chez les spécimens de l'Institut Pasteur de Paris de C. tritæniorhynchus et $C$. vishnui (1), que j'ai pu examiner (2), il y a un anneau complet autour de la trompe, mais la zone claire est beaucoup moins étendue sur la face ventrale.

(1) A propos de ces espèces consulter, entre autres :

Edwards (F. W.). - New synonymy in oriental culicida. Bull. entomol. res., IV, 1913-1914, p. 221.

Kirckpatrick (T. W.). - Mosquitos of Egypt., Gov. press, Cairo, 1925.

SÉGuY (E.). - Moustique de l'A frique Mineure, Paris, Lechevalier, 1924.

(2) Ces spécimens, qui font partie des collections de l'Institut Pasteur de Paris, m'ont été aimablement communiqués par le Prof. E. Roubaud. 
Palpes du mâle non annelés de blanc, une tache d'écailles claires sur la face inférieure du deuxième et troisième segments, près de leur base.

Thorax. - Mésonotum recouvert d'écailles brunâtres plus claires que chez C. tritæniorhynchus, les postérieures semblent plus claires que les autres. Sur les pleures, écailles blanches réparties en cinq groupes.

Ailes. - Chez le mâle, le pédicule de la fourchette antérieure (première cellule sub-marginale) est long. Les bases des fourchettes antérieure et postérieure ( $2^{\mathrm{e}}$ postérieure) sont au même niveau (fig. 2).

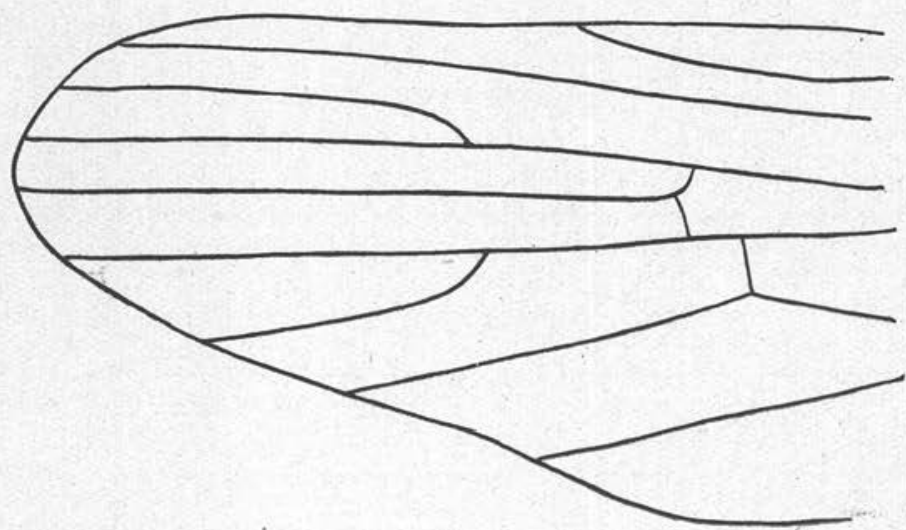

Fig. 2. - Aile du mâle.

Chez la femelle, pédicule de la première sub-marginale court. La distance qui sépare les deux transverses est égale à leur hauteur.

Pattes. - Non annelées de blanc. Face externe sombre, une tache blanche très nette au niveau des articulations fémoro-tibiale et tibio-tarsienne. La face interne de tous les articles, du fémur au cinquième segment du tarse, est recouverte d'une bande continue d'écailles marron clair.

Abdomen. - Presque entièrement dépourvu d'écailles sur la face dorsale des segments, sauf sur les bords où elles forment des taches blanches triangulaires, et sur la base du dernier segment où elles forment chez le mâle une bande complète.

Appareil génital mâle. - Sur le lobe sub-apical assez développé du premier article des forcipules, on trouve un premier groupe de trois appendices digitiformes, puis un second groupe comprenant 
deux appendices plus courts, dont l'un se termine en forme de harpon et deux palettes de taille inégale. En plus, une longue soie. Le second article est court, trapu, sans autre particularité (fig. 3).

Les appendices du $10^{\circ}$ sternite (fig. $4, a$ ) sont minces et présentent un bras basal long et grêle $(f)$. Les appendices chitineux du tube

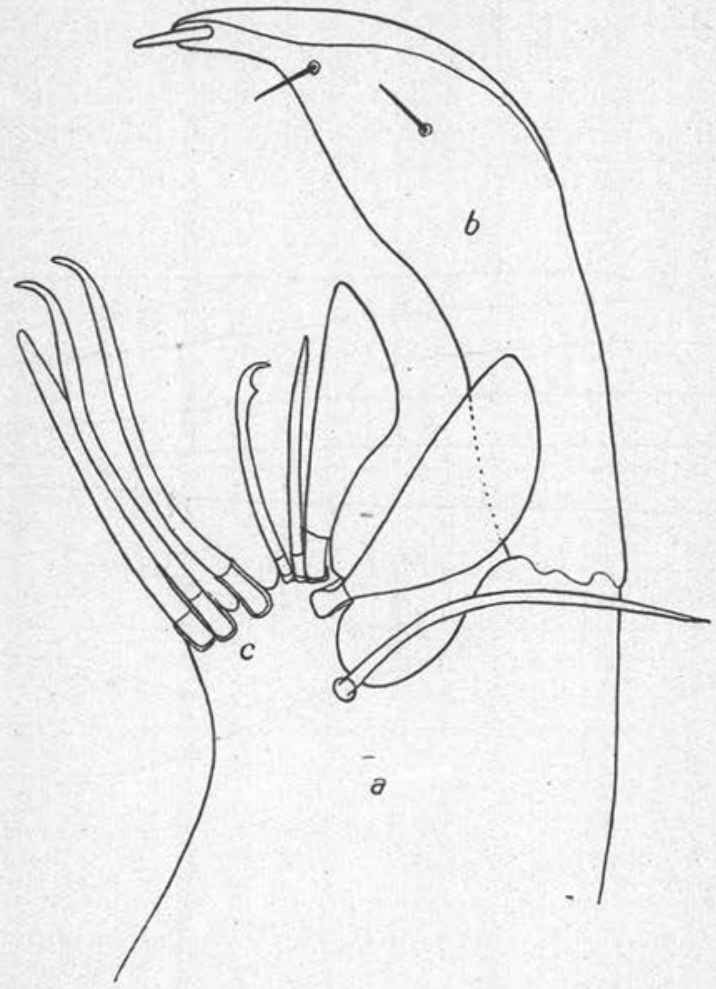

Fig. 3. - Un forcipule de l'appareil génital mâle : $a, 1$ er article ; $b, 2^{e}$ article ; $c$, lobe sub-apical avec ses appendices.

génital sont réduits à deux paires; une dent assez forte inclinée vers l'extérieur $(b)$ et un bras long et grêle dressé verticalement $(c)$, et s'élargissant à son sommet en forme d'éventail. Sur cette surface $(d)$, on trouve cinq crêtes longitudinales se terminant à la partie supérieure en forme d'épines qui ne dépassent pas le bord supérieur de cet appendice. En plus, à la base du mésosome, un bras cylindrique dirigé horizontalement en dehors $(e)$.

L'adulte ressemble done par ses caractères généraux au groupe 
des Culex comprenant C. tritæniorhynchus, C. vishnui, C. sitiens. Il s'en distingue nettement par la taille, par les caractères de la trompe, des pattes (tarses non annelés) et de l'appareil génital.

Habitat. - Côte occidentale de la Corse : Campo di Loro, près d'Ajaccio ; lit du Gravone (bras Nord), mare résiduelle avec conferves. Côte orientale : lit du Tavignano, près d'Aléria, même gîte.

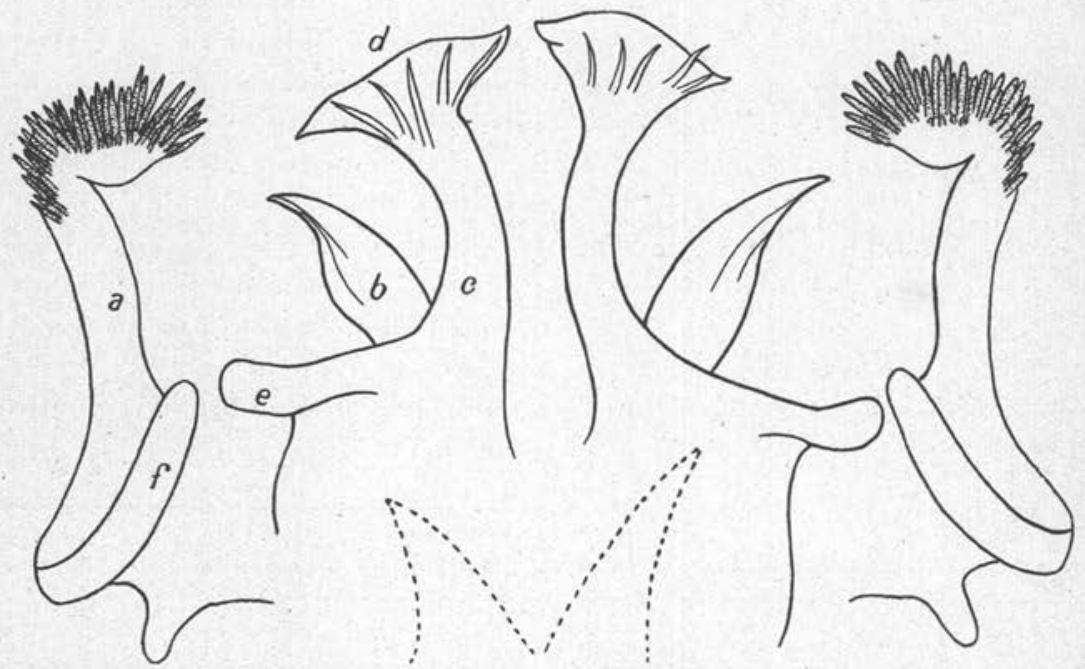

FIg. 4. - Appareil génital mâle (vu par face ventrale) : $a, 10^{e}$ sterníte; $b$ et $c$, les deux appendices chitineux, dorsal et ventral du tube génital; $d$, sommet du 2. appendice (ventral); $e$, appendice basal cylindrique; $f$, bras basal du $10^{*}$ sternite.

\section{RÉSUMÉ}

Nous décrivons dans cette note une espèce nouvelle de Culex (C. brumpti) trouvé en Corse; elle se rapproche du groupe des moustiques orientaux à trompe annelée (groupe du Culex tritæniorhynchus), mais les caractères de l'adulte et de la larve sont suffisamment typiques pour permettre de l'individualiser nettement.

Laboratoire de Parasilologie de la Facullé de Médecine de Paris 